Article

\title{
Load Transfer Path Search and Its Evaluation between Networks in Consideration of the Mobile Energy Storage of Electric Vehicles
}

\author{
Dongyu Mao ${ }^{1, *}$, Xueliang Huang ${ }^{1}$, Zhong Chen ${ }^{1}$, Yang Lv $^{2}$, Jiang Tian ${ }^{2}$ and Zexin Yang ${ }^{1}$ (D) \\ 1 School of Electrical Engineering, Southeast University, Nanjing 210096, China; xlhuang_ee@seu.edu.cn (X.H.); \\ chenzhong_seu@163.com (Z.C.); zexin_yang@seu.edu.cn (Z.Y.) \\ 2 State Grid Jiangsu Electric Power Co., Ltd., Suzhou Power Supply Branch, Suzhou 215004, China; \\ tmac132180@163.com (Y.L.); zzqwxyd@126.com (J.T.) \\ * Correspondence: maodongyu@seu.edu.cn
}

Citation: Mao, D.; Huang, X.; Chen, Z.; Lv, Y.; Tian, J.; Yang, Z. Load Transfer Path Search and Its Evaluation between Networks in Consideration of the Mobile Energy Storage of Electric Vehicles. World Electr. Veh. J. 2021, 12, 210. https:// doi.org/10.3390/wevj12040210

Academic Editor: Zonghai Chen

Received: 1 September 2021

Accepted: 15 October 2021

Published: 25 October 2021

Publisher's Note: MDPI stays neutral with regard to jurisdictional claims in published maps and institutional affiliations.

Copyright: (c) 2021 by the authors. Licensee MDPI, Basel, Switzerland. This article is an open access article distributed under the terms and conditions of the Creative Commons Attribution (CC BY) license (https:/ / creativecommons.org/licenses/by/ $4.0 /)$.

\begin{abstract}
Load transfer is an important way of restoring a power supply after equipment failure or maintenance. However, current methods ignore cooperation between networks and users, and they also fail to take into account the vehicle-to-grid (V2G) potential of electric vehicles (EVs). In this paper, a load transfer scheme between transmission and distribution networks is proposed, considering the mobile energy storage capacities of electric vehicles. First, the mobility characteristic and the available discharge capacity of EVs are analyzed on the basis of the parking generation rate. Then, the breadth-first algorithm is used to search the load transfer paths within and between stations, and an edge matching method is proposed to realize the conversion between networks with different voltage levels. Lastly, the optimal combination weighting method was adopted to combine subjective and objective index weights and to evaluate power supply paths. The effectiveness of the proposed scheme is validated in a case composed of an IEEE 30-node network and an IEEE 57-node network, with four typical scenarios.
\end{abstract}

Keywords: load transfer; V2G; parking generation rate; breadth-first search; edge matching; optimal weighting method

\section{Introduction}

Load transfer is an important method for restoring a power supply or adjusting loads, enabling power flow with an even distribution and preventing grid congestion. Distribution networks contain a large number of contact and sectional switches, which can be used to achieve fault clearing and load transfer by adjusting the switching state. The main application scenarios for load transfer are (1) increased load leading to heavy equipment load or overload, (2) equipment being out of operation due to failure or planned maintenance, and (3) an increase in power from renewable energy needing to be absorbed locally.

At present, the operation scheme is still provided by dispatchers on the basis of their previous experience or preferences, which makes it impossible to ensure scheme optimality. Due to the uncertainty of new energy outputs and the randomness of electric vehicle loads, load transfer is becoming an increasingly relevant issue. With the increasing popularity of EVs, their total energy storage capacity is increasing. The continuous advancement of (V2G) technology enables EVs to participate in load transfer in distributed generation, playing a role in peak shaving and valley filling.

Researchers have presented many solutions to this problem, taking approaches that can be divided into four categories. Those in the first group [1-4] adopted a heuristic search algorithm, taking the tie switch as a variable and calculating the load transfer scheme. This makes it possible to reduce the solution space and offers good generality but cannot guarantee the optimality of the solution. Those in the second group [5-7] used a 
stochastic optimization algorithm. This makes it possible to solve multi-objective problems, and the model is relatively complete, but the calculation time is too long to achieve the speed required for online application, thus making it unsuitable for large-scale networks. Researchers in the third group [8,9] used the combination of a heuristic algorithm and stochastic optimization, optimizing the scheme while ensuring fast calculation. Those in the fourth group [10] used the expert system method. After establishing an expert database, a fault recovery operation scheme can be automatically generated, offering good real-time performance and the ability to be applied in large-scale networks.

However, the above algorithms ignore cooperation between networks and users, and they do not take into account the discharge capacity of EVs. In this paper, an auxiliary decision-making method for load transfer based on path search and evaluation is proposed for application in scenarios of heavy overload, maintenance, and operation mode switching of the distribution network. This paper proposes an innovative load transfer and evaluation method between transmission and distribution networks that considers the mobile energy storage of EVs. Compared with existing methods, the main contributions of this paper are fourfold: (1) EV clusters are considered as a power supply source, and the parking generation rate is applied in order to build a model of their discharge capacity; (2) an innovative inter-/intra-station search algorithm and a matching algorithm are proposed, which are used to search the power supply path of power generation for users across voltage levels and management systems; (3) the optimal combination weighting method is used to evaluate transfer paths; (4) examples of the main and distribution networks are constructed, along with several typical scenarios, in order to prove the effectiveness of the proposed method.

\section{Mobile Energy Storage Capacity of EVs}

V2G means that EVs can charge and discharge to the power grid, acting as energy storage units when they are parked. This study transforms the control object from a single EV to a regional cluster of EVs. The movement of single vehicles is indirectly reflected in the parking characteristics of vehicle groups in the region, and it can be described by the parking generation rate (PGR).

As illustrated in Figure 1, an area may be divided into several zones (zones 1, 2, . , n), such as commercial zones and residential zones. The PGR of the $k$-th area is recorded as $f_{k}$. Electric vehicles can park or move freely from one zone to another, and their movement behavior is characterized by the PGR of each zone. Considering that vehicles are moving in and out of the area all the time, the total number of electric vehicles in the area varies at any given time [11]. The process of calculating the parking production rate is depicted in Figure 1.

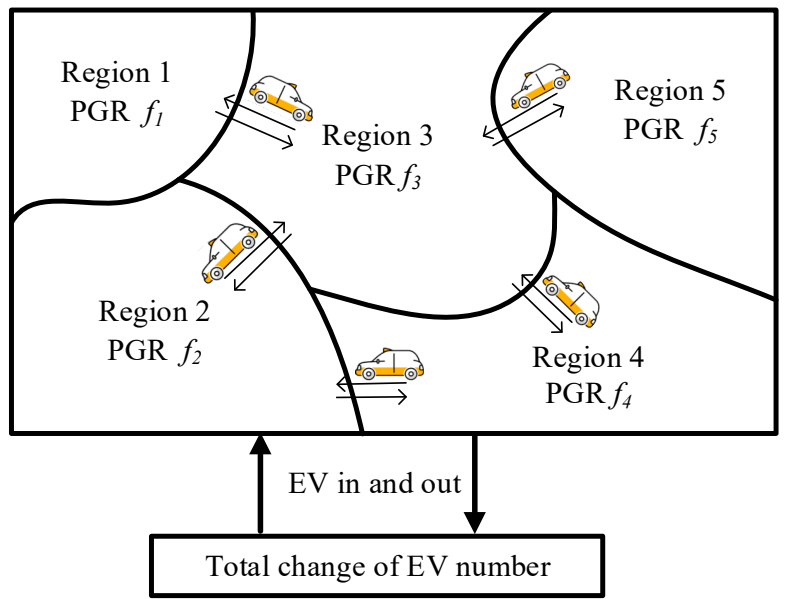

Figure 1. Schematic diagram of mobility of EVs. 


\subsection{Parking Generation Rate}

Parking generation rate is an important indicator of regional parking demand. A large number of vehicles will park in the area when the area has a high PGR, while vehicles will leave when the PGR is low. In the traditional PGR rate model, the parking demand of an area is equal to the parking generation rate of the area multiplied by the building area of the area. The calculation formula is

$$
D_{k}=f_{k} S_{k}
$$

where $D_{k}$ is the parking demand in area $k, f_{k}$ is the PGR of area $k$, and $S_{k}$ is the acreage of area $k . D_{k}$ can also represent the number of occupied parking spaces, while $S_{k}$ is the total number of parking spaces in area $k$.

The overall idea of EV mobility modeling is to obtain PGR curves based on historical statistics and to compute typical data for EV numbers parked in each time period. At the beginning of each period, the state of EVs is dynamically adjusted by the difference between the current parking quantity and the parking generation rate model calculation results.

At each state update time, the difference $\left(D_{P}\right)$ between the regional parking demand obtained by PGR model and the actual number of electric vehicles parked in the current area is calculated. If $D_{P}>0$, vehicles on the move are randomly selected to park in the area; if $\mathrm{D}_{\mathrm{P}}<0$, vehicles whose state of charge (SOC) meet the departure requirements will be randomly selected to leave. Then, the status signs of each EV are updated to make them conform to the travel characteristics of each region. The driving record of an EV can be obtained from its status mark. The process of updating the moving state of EVs on the basis of PGR is shown in Figure 2.

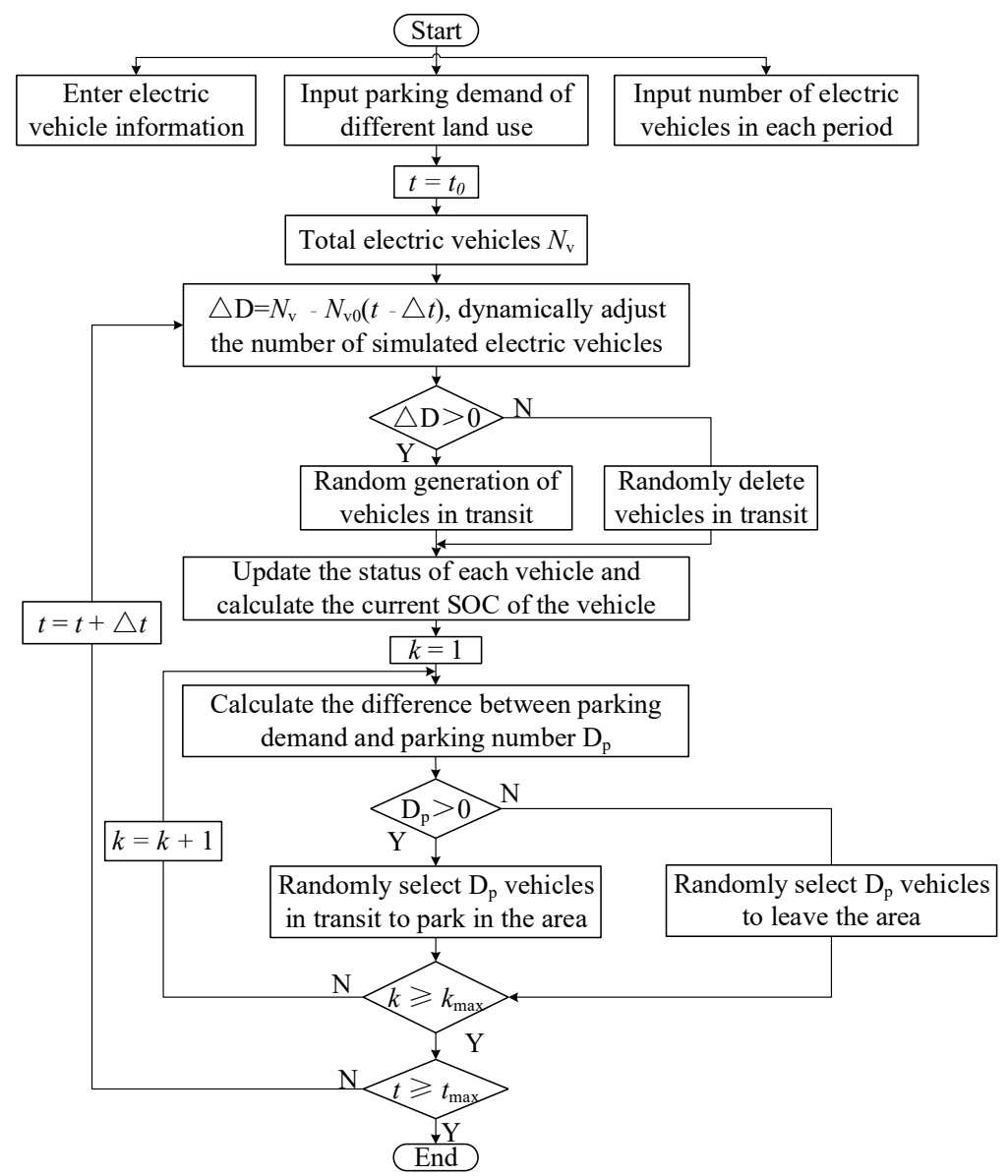

Figure 2. EV movement status update process based on PGR. 
Since the focus of this paper is to establish a charge-discharge model of EVs, the PGR data were taken from [12], where they were obtained according to the statistics of the actual traffic and parking space information of a city.

\subsection{Mobile Energy Storage Model of EV}

This paper describes the EV status according to [10], as outlined below.

(1) $\quad F_{\text {area }}(n)$ is the area status of the $n$-th $\mathrm{EV}$, and its calculation formula is

$$
F_{\text {area }}(n)=\left\{\begin{array}{l}
i, \text { EV parked in area } i \\
0, \text { EV is on the move }
\end{array} .\right.
$$

(2) $S_{\mathrm{OC}}$ is the charge state of the battery.

(3) $Q$ is the battery capacity, in units of kWh.

(4) $\bar{v}$ is the average speed of the EV, in units of $\mathrm{km} / \mathrm{h}$.

(5) $F_{\mathrm{P}}$ is the parking sign of the electric vehicle, and its calculation formula is

$$
F_{\mathrm{P}}=\left\{\begin{array}{l}
1, F_{\text {area }} \neq 0 \\
0, F_{\text {area }}=0
\end{array} .\right.
$$

(6) $W_{100}$ is the power consumption per $100 \mathrm{~km}$, in units of $\mathrm{kWh}$.

(7) $S_{\max }$ and $S_{\min }$ are the upper and lower limits of $S_{\mathrm{OC}}$ when the EV is connected to the power grid.

The energy storage consumption rate of electric vehicles is

$$
v_{\mathrm{SOC}}=\left(1-F_{\mathrm{P}}\right) \bar{v} \frac{W_{100}}{100 Q} .
$$

The charging state of the $n$-th EV in period $t$ is

$$
S_{\mathrm{OC}}(t, n)=S_{\mathrm{OC}}(t-\Delta t, n)-v_{\mathrm{SOC}}(n) \Delta t,
$$

where $\Delta t$ is the time interval of the status update, and $S_{\mathrm{OC}}(t, n)$ is the state of charge of the $n$-th EV in period $t$.

In the parking state, EVs participate in V2G, and the energy storage calculation formula is

$$
S_{\mathrm{OC}}(t, n)=S_{\mathrm{OC}}(t-\Delta t)+\frac{P(t, n) \Delta t}{Q},
$$

where $P(t, n)$ is the charging and discharging power of the $n$-th vehicle participating in the grid interaction in period $t$.

Thus, the discharge capacity of EV clusters in each region to the power grid can be obtained.

\section{Load Transfer Path Search}

Load transfer is used to resolve problems relating to power line overloads, equipment overhaul, or operation mode changes. The power system dispatchers formulate reasonable solutions to these expected fault sets. In order to achieve the goal of integrated dispatching of networks and users, it is necessary to search the user range affected by the fault, and then search the potential power supply path for important users. Taking the maintenance of certain equipment as an example, downstream users are first searched along the power transmission direction, which is the outage range that may result from its exit from operation. Then, the possible power supply path is searched against the power transmission direction, in order to obtain the load transfer scheme for users in that range.

However, power networks adopt distributed management, while main and distribution networks are stored in different systems. For example, in some areas, the distribution 
network model and the data are stored in the D5200 system, while the network model and data for a higher voltage level are stored in the D5000 system. The two systems are not directly interconnected, which makes it difficult to form power supply paths from generators to users through different voltage levels.

In this section, a method of electrical network path search combining different layers and systems is proposed. When searching paths in a particular system, stations are regarded as basic nodes and power lines are considered as connecting lines. When traversing to a basic node, installations inside the station are searched. After completing a topological search within the system, a corresponding inter-system path search algorithm based on edge matching is proposed.

\subsection{Power Supply Path Search Algorithm inside a System}

The typical topology inside a station is shown in Figure 3a. Components other than wires are regarded as unit nodes, and wires are regarded as branches when modeling. The typical topology between stations is shown in Figure 3b. Power plants and stations are regarded as unit nodes, and tie lines between plants and stations are considered as branches connecting nodes during modeling [13].

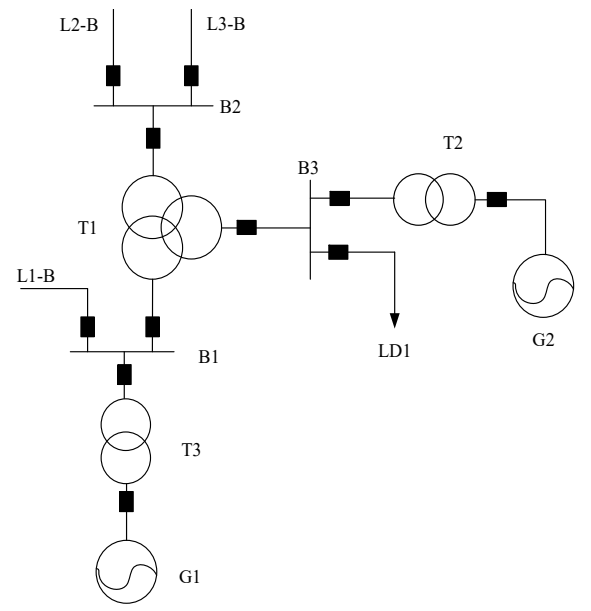

(a)

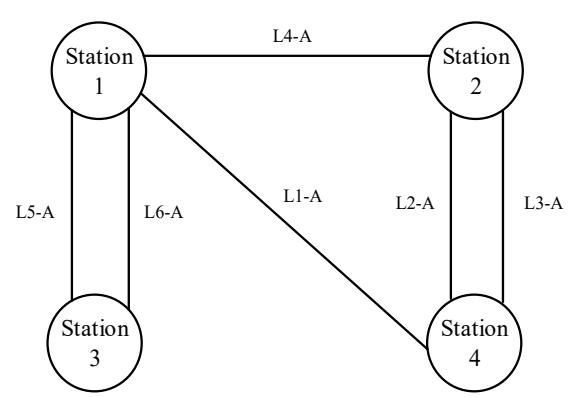

(b)

Figure 3. Typical connection diagram: (a) station wiring diagram; (b) inter-station wiring diagram.

Taking the defective equipment inside the station as the starting node, a breadth-first width search (BFS) inside the station is initiated until all outgoing lines are traversed. Then, depending on the connection relationship between the stations and taking the outgoing line as the starting point, a BFS is conducted between the stations. For inter-station traversal, stations connected to the upper layer are considered to be a new layer. Assuming that the search advances to layer $N$, there are $m$ stations in this layer, namely, $S T_{n, 1}, S T_{n, 2}, \ldots, S T_{n, m}$, and the tie lines between each station on layer $N-1$ are $W_{n, 1}, W_{n, 2}, \ldots, W_{n, k}$. At this point, inter-station traversing is stopped, the tie lines are taken as starting points, and the BFS is initiated inside the station. The searched outgoing lines of layer $N$ in $W_{n+1}$ are saved. If all stations in layer $N$ complete intra-station traversal and $S$ outgoing lines are found, the contact relationship between layer $N$ and layer $N+1$ is determined, and the starting points of the next round of the inter-station search can be determined accordingly. The intra-station traversal is continued for layer $N+1$, before being transferred to the inter-station traversal to layer $N+2$. The iteration continues until all nodes advance to the ultimate layer.

The path search algorithm for the system is shown in Figure 4. 


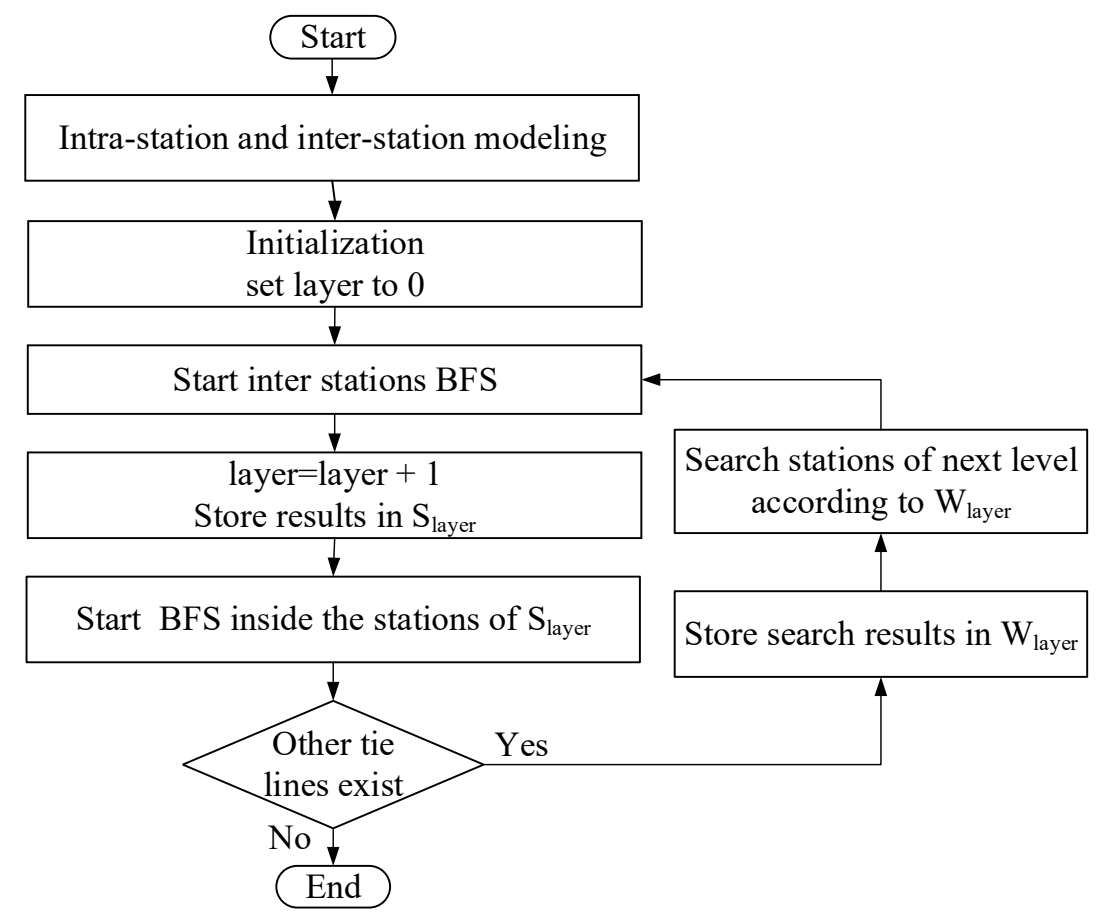

Figure 4. Path search algorithm between and inside stations.

\subsection{Power Supply Path Search Strategy between Systems}

In order to realize the comprehensive consideration of the load transfer scheme, the idea of main and distribution network joint scheduling proposed in [11] can be adopted. Different voltage levels are used for power transmission, and power grids of different voltage may be operated and managed by different systems; hence, it is necessary to solve the problem of cross-system path search.

The same power station or power line may have different names in the different dispatching systems. Therefore, in this paper, a similarity function is proposed to realize edge matching when the main and distribution network conversion is involved in the path search. As shown in Equation (7), three similarities are defined, i.e., the voltage similarity, the current similarity, and the name similarity, and the minimum value of the three $S_{i, j}$ values is taken as the final similarity between node $i$ and node $j$.

$$
S_{i, j}=\min \left\{1-\left|\frac{V_{j}-V_{i}}{V_{i}}\right|, 1-\left|\frac{I_{j}-I_{i}}{I_{i}}\right|, \frac{A_{j}}{T_{i}+\alpha}\right\}, j \in R,
$$

where $i$ is the number of nodes to be matched in the system, $j$ is the number of nodes in another system, $V$ is the node voltage and $I$ is the node current, $T_{i}$ is the word number of the $i$-th line, $A_{j}$ is the word number with the same description for the $j$-th outgoing line, $\alpha$ is the minimum, and $R$ is the set of all lines in another system.

When searching for the fault range, if an equipment failure occurs in the distribution network, load nodes are searched along the active power flow direction to obtain the affected area $N_{P S}$. If the fault occurs in the main network, the affected node set in the main network $N_{Z S}$ is searched from the faulty equipment, and then the corresponding node set $N_{Z P}$ in the corresponding distribution network is obtained via the edge matching method. Starting from $N_{Z P}$, the influence range of $N_{P S}$ in the distribution network is sought in the direction of the active power stream.

In this study, when searching power supply paths starting from $N_{P S}$, and taking the transformer as the end point, the power supply path in the distribution network is searched against the active power flow to obtain the set of possible power supply nodes, and then the set of nodes in the main network $N_{P Z}$ is obtained via the edge matching method. Starting with $N_{P Z}$ and taking transformers as the end point, the power supply paths in the main 
network are obtained by searching against the active power flow. The search strategy is illustrated in Figure 5.

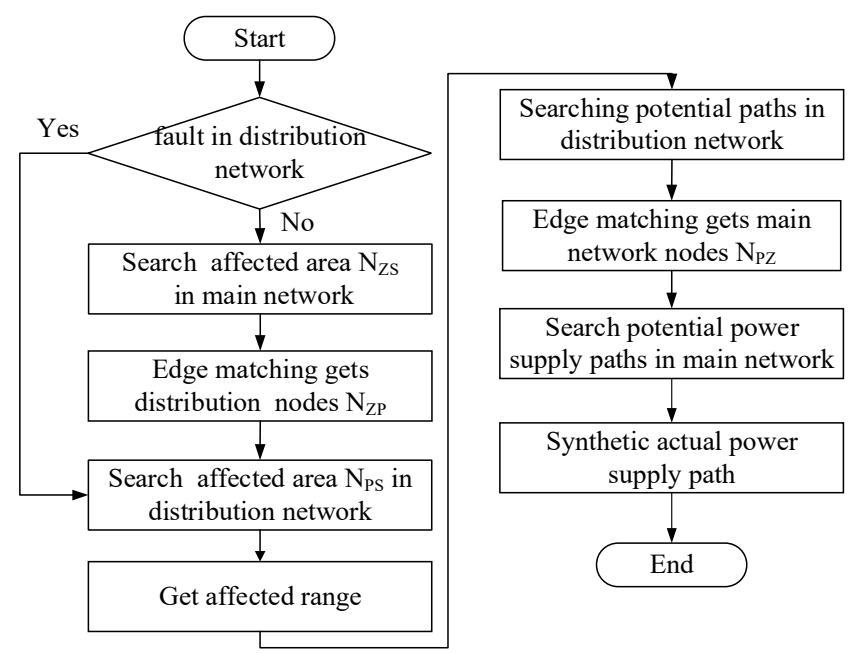

Figure 5. Power supply path search strategy.

\section{Path Evaluation Algorithm}

In order to evaluate transfer paths from multiple perspectives and improve the rationality and the applicability of the evaluation method, this study first determines the evaluation index set, while the participation rate of EVs is also taken into account. The expert scoring method is used to determine the subjective weight of the index while the entropy weight method is used to determine the objective weight, and then the optimal combination weight is used to determine the final weight of the index [14].

\subsection{Index Set}

This study considers the security and economy of the power grid operation, taking into account the new energy consumption, the participation of electric vehicles, etc. The six indices shown below were obtained.

- Load rate change of main transformer:

$$
N_{1}=\frac{S_{N L}}{S}-\frac{S_{L}}{S},
$$

where $S$ is the rated capacity of the main transformer, $S_{N L}$ is the apparent power of the main transformer with extra load, and $S_{L}$ is the apparent power of the main transformer before the fault [15].

- Remote node voltage deviation:

$\mathrm{N}_{2}$ is the voltage deviation of the load node farthest from the main transformer after load transfer, with units of p.u.

- Current load rate change:

$N_{3}$ is the difference in current load rate on the load transfer line.

$$
N_{3}=\frac{I_{N L}}{I_{N}}-\frac{I_{L}}{I_{N}},
$$

where $I_{N}$ is the rated current of the load transfer line, $I_{L}$ is the original current on the load transfer line, and $I_{N L}$ is the current on the line after load transfer.

- Switch operation times:

$N_{4}$ is the number of tie switch operations during load transfer, and is dimensionless. 
- Additional network loss rate:

$$
N_{5}=\frac{P_{l}-P_{r l}}{S_{l}},
$$

where $P_{l}$ is the network loss of all lines with extra load power points after load transfer, $P_{r l}$ is the network loss of all lines before the power failure, and $S_{l}$ is the rated transmission capacity.

- $\quad$ EV participation rate:

$$
N_{6}=\frac{L_{t r}}{C_{E V}}
$$

where $C_{E V}$ is the EV capacity, and $L_{t r}$ is the load transferred to EVs (in units of MW).

\subsection{Optimal Combination Weighting Method}

There are major differences between the subjective weighting method and objective weighting method. In order to make the scheme evaluation more scientific, the two methods can be combined according to the optimal combination weighting method so that the final index weight coefficients can take into account both the subjectivity of the individuals and the objectivity of the data [16].

\subsubsection{Subjective Weight}

The expert scoring method was used to calculate the subjective weights of indices in Section 4.1, based on the subjective judgment of experts or decision makers. The rationality of weight setting can be explained, but different experts may have different opinions regarding the same index. In this paper, the mean value of the results of the expert scoring method was taken as the subjective weight.

\subsubsection{Objective Weight}

The entropy weight method (EWM) was used to calculate objective values. The objective weighting method obtains the corresponding weight on the basis of the values of the indices themselves, which has strong objectivity but lacks explanatory power, and the calculated weight may be contrary to reality.

First, the data are standardized; then, the calculation method of the negative index is

$$
Y_{i j}=\frac{\max \left(x_{i}\right)-x_{i j}}{\max \left(x_{i}\right)-\min \left(x_{i}\right)},
$$

where $x_{i}$ is the $i$-th evaluation index, and $x_{i j}$ is the value of the $j$-th index of the $i$-th scheme.

Next, the information entropy is calculated according to Equation (13), and objective index weights are determined using Equation (14).

$$
\begin{gathered}
E_{j}=-\ln (n)^{-1} \sum_{i=1}^{n} p_{i j} \ln p_{i j}, P_{i j}=\frac{Y_{i j}}{\sum_{i=1}^{n} Y_{i j}}, \\
W_{i}=\frac{1-E_{i}}{k-\sum E_{i}}(i=1,2, \ldots k),
\end{gathered}
$$

where $Y_{i j}$ is the normalized value of each index data point, $n$ is the evaluation index scheme number, and $k$ is the evaluation index number.

\subsubsection{Optimal Combination Weighting}

In order to make the evaluation scheme more scientific, the optimal combination weight method was applied to obtain combination weights. For power supply paths, it 
was assumed that there are $n$ evaluation indices and $s$ index weighting methods. Then, the $k$-th weighting method can be expressed as

$$
W^{k}=\left(w_{1}^{k}, w_{2}^{k}, \ldots w_{n}^{k}\right), k=1,2, \ldots, s .
$$

Thus, the optimal weight is expressed as

$$
W^{0}=\left(w_{1}^{0}, w_{2}^{0}, \ldots w_{n}^{0}\right), \sum_{j=1}^{n} W_{j}^{0}=1 .
$$

The optimization model constructed in this paper aims to minimize the deviation between the combined weight and the known index weight. This model can take into account all the known weights. In order to construct the optimal model, it is necessary to determine the deviation between the combination weight vector $W^{0}$ and the $k$-th weighting method; the calculation formula is described below.

The optimization model is

$$
\left\{\begin{array}{l}
\min \sum_{k=1}^{s}\left\|W^{0}-W^{k}\right\|^{2}=\min \sum_{k=1}^{s} \sum_{j=1}^{n}\left(w_{j}^{0}-w_{j}^{k}\right)^{2} \\
\text { s.t. } \sum_{j=1}^{n} w_{j}^{0}=1
\end{array},\right.
$$

where $W^{k}$ represents the $k$-th weighting method, and $W^{0}$ represents the optimal combination weight vector. The Lagrange function can be constructed to solve the problem, and an optimal weight coefficient can be obtained.

$$
L\left(w_{j}^{0}, \lambda\right)=\sum_{k=1}^{s} \sum_{j=1}^{n}\left(w_{j}^{0}-w_{j}^{k}\right)^{2}+\lambda\left(\sum_{j=1}^{n} w_{j}^{0}-1\right)
$$

If we obtain the first partial derivatives of $w_{j}^{0}$ and $\lambda$ and set them to zero, then the combination weight vector can be obtained using the above formula [17].

\section{Case Studies}

In this paper, a combined IEEE calculation example was constructed, as shown in Figure 6a. There were 30 plants and stations in the main network calculation example, numbered 1-30, and 57 plants and stations in the distribution network calculation example, numbered 1-57. Nodes 26, 21, 24, and 14 of the main network were regarded as step-down transformers, and nodes 1, 2, 3, and 6 of the distribution network were connected in order. EV clusters were connected at nodes 8, 9, and 12 in the distribution network. Black dots in the figure represent equivalent load plants and stations. The corresponding node power was adjusted to balance the whole network power. The regional division of nodes in the distribution network is described in Table 1 , and typical load curves in different areas are shown in Figure 6b. 


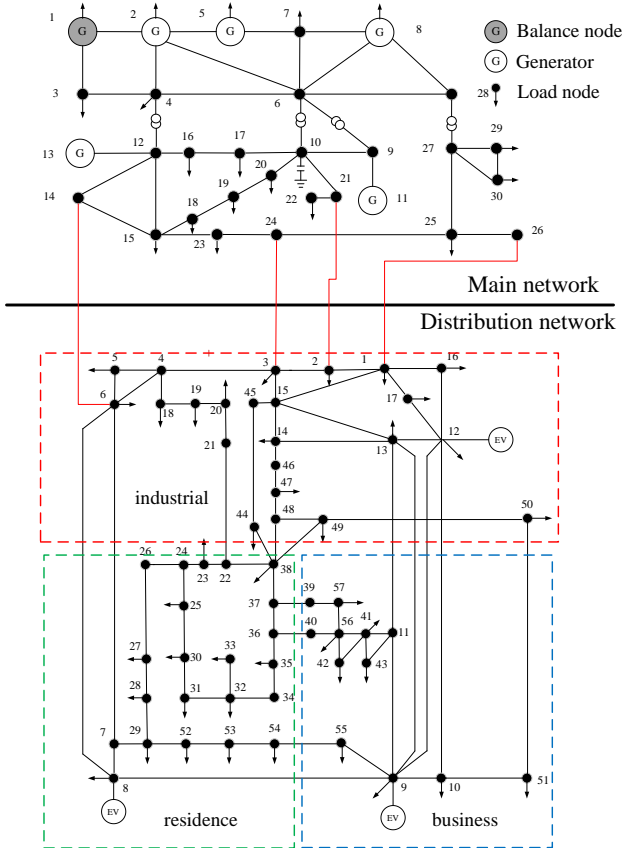

(a)

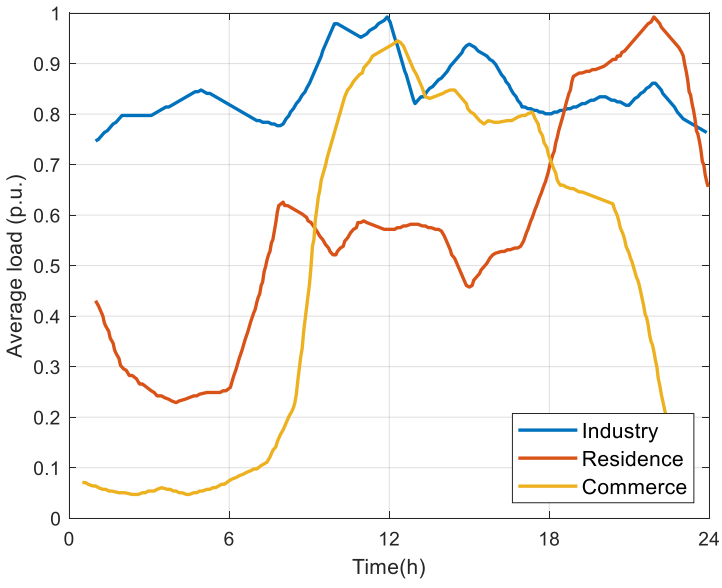

(b)

Figure 6. Example diagram and load data: (a) diagram of calculation example; (b) typical load curve of each area.

Table 1. The 57-node calculation case partition $(20 \mathrm{kV})$.

\begin{tabular}{cr}
\hline Region & Node \\
\hline Industry & 1234561213141516171819202144454647484950 \\
Business & 91011394041424351555657 \\
Resident & 782223242526272829303132333435363738525354 \\
\hline
\end{tabular}

\subsection{Mobility Characteristics of EV}

1. Non-working days

The typical parking generation rate curve for each area on non-working days is shown in Figure 7a. Compared with working days, the parking characteristics of vehicles in residential areas and industrial and commercial areas at night on non-working days are basically unchanged, and the difference is mainly apparent in the daytime. The number of vehicles going out of residential areas during the day is reduced, and the parking demand is increased. The number of vehicles arriving in industrial and commercial areas during the day is also reduced; hence, the parking demand is reduced.

\section{Weekdays}

Figure $7 \mathrm{~b}$ shows the typical parking generation rate curve for each area on a working day. The parking rate in a residential area is close to 1, indicating that there are several vehicles parked at night. Most vehicles leave at about 7:00 a.m. After 6:00 p.m., a large number of vehicles return to the residential area. The parking rules of industrial and commercial areas are complementary to those of residential areas. The number of parking vehicles at night is lower, and these are usually commuter vehicles, but there are many parked vehicles in the daytime, and the peak of parking demand appears at about 12:00 p.m. Changes in parking rates can reflect the movement trend of vehicles. 


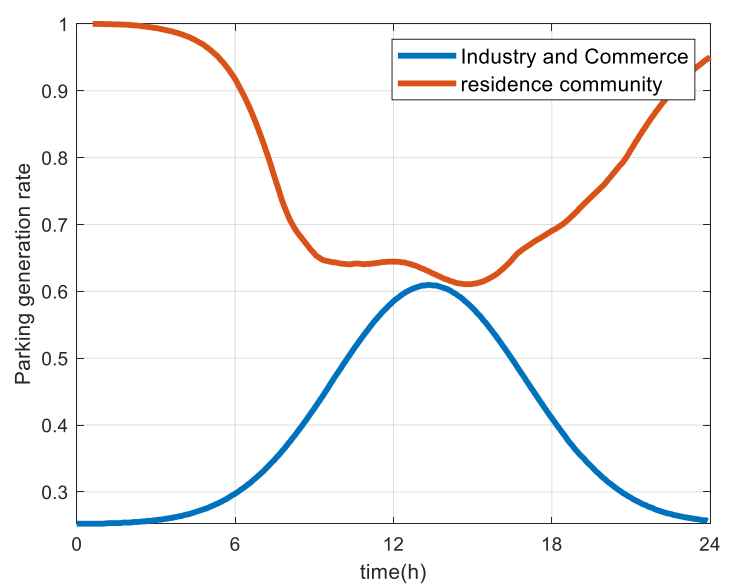

(a)

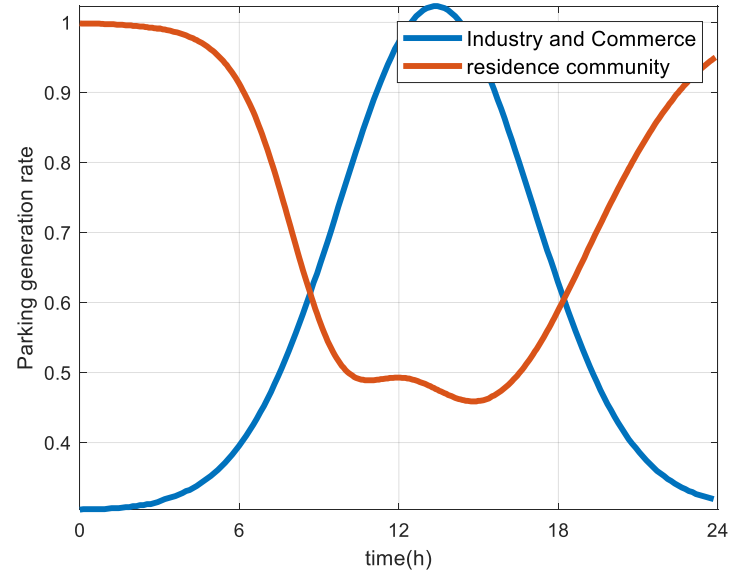

(b)

Figure 7. EV parking generation rate: (a) non-working day PGR; (b) weekday PGR.

According to the parking generation rate, the available energy storage capacity of electric vehicles in different time periods in each area can be obtained. The available capacity curves of EVs in each region are shown in Figure 8. It can be seen that the trend basically conforms to the trend of the parking generation rate.

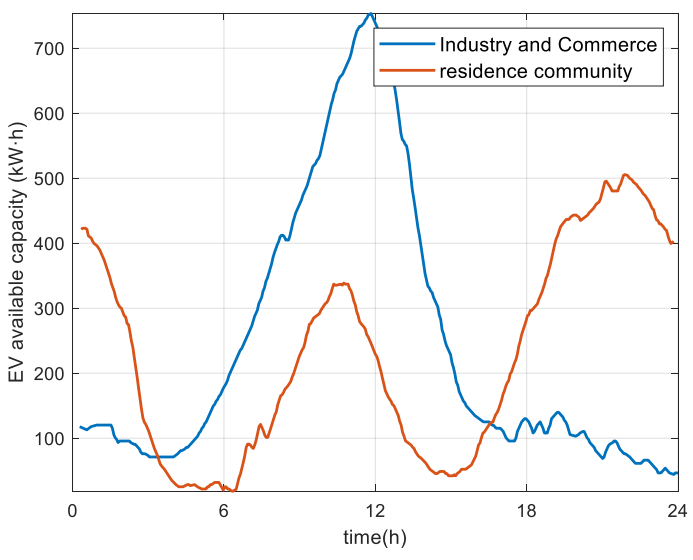

(a)

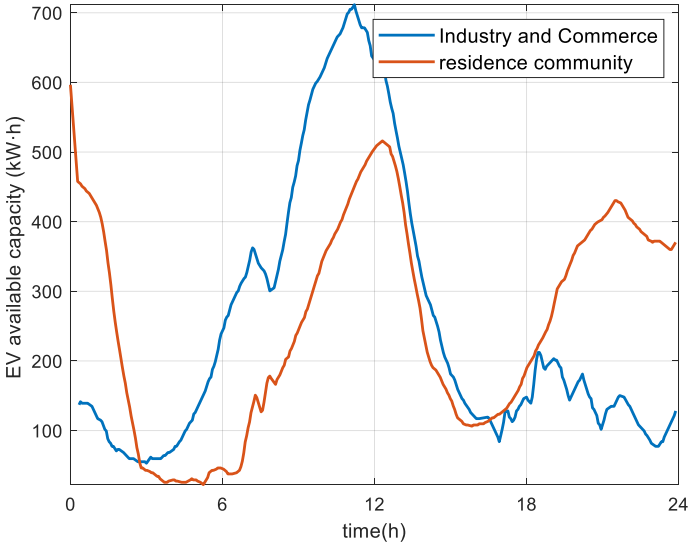

(b)

Figure 8. Electric vehicle discharge capacity: (a) non-working day EV capacity; (b) weekday EV capacity.

\subsection{Path Search and Evaluation}

The nodes in Figure 6a represent the plant and the station in the power system. The 30-node network was the main network, which was managed in system 1, and the 57-node network was the distribution network, which was managed in system 2 . It was assumed that the edge matching method mentioned in Section 3.2 was carried out, and the connection relationship between the networks is as shown in the figure. Since the focus of this study was the load transfer scheme considering EV V2G and its evaluation, the fault range and potential power supply paths between plants and stations were searched.

The distribution network fault was set as the 1\# transformer in node 12 quit operation. Since the fault occurred in the distribution network, it was only necessary to search along the active flow direction when searching the fault range. The fault influence range was found to be only node 50 . Then, the power supply path search was initiated, and the switch action time limit was added. The potential path search results in a distribution network, and standardized assessment results without considering EV discharge are shown in Table 2. Column 2 represents the potential power supply paths composed of node numbers in the distribution network. Columns 3-8 represent the evaluation results of the indices N1-N6 
proposed in Section 4.1. The last column is the path evaluation result after the optimal combination weight calculation.

Table 2. Distribution network route and evaluation $(20 \mathrm{kV})$.

\begin{tabular}{ccccccccc}
\hline No. & Path & N1 & N2 & N3 & N4 & N5 & N6 & Evaluation Results \\
\hline 1 & {$[50,49,13,15,1]$} & 1.00 & 0 & 0 & 0 & 0 & 0 & 0.33 \\
2 & {$[50,49,13,15,3]$} & 0.97 & 0.35 & 0.79 & 0.00 & 0.95 & 0 & 0.5 \\
\hline
\end{tabular}

The search results considering electric vehicle discharge are shown in Table 3, which provides a certain reference for power grid operators and dispatchers. Schemes 1, 4, and 5 transfer the load of node 50 to the electric vehicle cluster in the network, and schemes 2 and 3 transfer it to other transformers in the network.

Table 3. Distribution network route and evaluation $(20 \mathrm{kV})$.

\begin{tabular}{ccccccccc}
\hline No. & Path & N1 & N2 & N3 & N4 & N5 & N6 & Evaluation Results \\
\hline 1 & {$[50,49,13,9]$} & 0.96 & 1 & 0.9 & 1 & 0.98 & 0.05 & 0.81 \\
2 & {$[50,49,13,15,1]$} & 1 & 0 & 0 & 0 & 0 & 0 & 0.16 \\
3 & {$[50,49,13,15,3]$} & 0.97 & 0.35 & 0.79 & 0 & 0.95 & 0 & 0.47 \\
4 & {$[50,51,10,9]$} & 0.96 & 0.55 & 1 & 1 & 0.99 & 0.05 & 0.74 \\
5 & {$[50,51,10,12,9]$} & 0 & 0.07 & 0.94 & 0 & 1 & 1 & 0.46 \\
\hline
\end{tabular}

It can be seen that, compared with Table 2, the transfer path number increased to 5 when considering EV discharge, and scores of the new paths were higher than the original paths. The five schemes in Table 3 had their own advantages and disadvantages according to different evaluation indices; however, after the optimal weight calculation, the final evaluation result of scheme 1 was the best. Scheme 1 had fewer switches, a closer electrical distance, a smaller terminal voltage offset, and smaller additional network loss, when the EV cluster was used to carry part of the load. It can be seen that the final evaluation results are more in line with objective reality, reflecting the advantages of the optimal weight comprehensive evaluation from the subjective and objective perspectives.

In this paper, four different typical scenarios were considered in Section 5.2, and the evaluation results are shown in Table 4.

Table 4. Evaluation results for typical scenarios $(20 \mathrm{kV})$.

\begin{tabular}{ccccccc}
\hline No. & Node & Working Day & Weekday Night & Holiday & Holiday Night & Average \\
\hline 1 & {$[50,49,13,9]$} & 0.75 & 0.73 & 0.81 & 0.79 & 0.77 \\
2 & {$[50,49,13,15,1]$} & 0.33 & 0.33 & 0.28 & 0.41 & 0.34 \\
3 & {$[50,49,13,15,3]$} & 0.3 & 0.24 & 0.42 & 0.49 & 0.36 \\
4 & {$[50,51,10,9]$} & 0.72 & 0.7 & 0.77 & 0.83 & 0.76 \\
5 & {$[50,51,10,12,9]$} & 0.48 & 0.36 & 0.33 & 0.31 & 0.37 \\
\hline
\end{tabular}

The results show that scheme 4 was the best on holiday nights, while scheme 1 was the best in the other three scenarios. Taking the average value of the evaluation results of different scenarios for each scheme, it can be concluded that scheme 1 was the comprehensively optimal scheme. Scheme 4 was second only to scheme 1 , and the difference was relativity small; thus, it can be considered an alternative choice.

After completing the path search of the distribution network, the search of the main network was commenced. The main network path search results and evaluation results for different scenarios are shown in Table 5. By combining the path search and evaluation results of the main network and the distribution network, the power supply path scheme connecting the main network, distribution network, and users could be obtained. 
Table 5. Main network path and evaluation (110 kV).

\begin{tabular}{ccccccccc}
\hline No. & Node & N1 & N2 & N3 & N4 & N5 & N6 & Evaluation Results \\
\hline 1 & {$[26,25,27]$} & 1 & 0.02 & 0.22 & 0 & 0.21 & 0 & 0.3 \\
2 & {$[24,23]$} & 0 & 1 & 1 & 1 & 1 & 0 & 0.86 \\
5 & {$[24,25,27]$} & 0.58 & 0 & 0 & 0 & 0 & 0 & 0.12 \\
\hline
\end{tabular}

As the capacity for load transfer was far less than the load of the nodes in the main network, the difference in power supply paths of different main networks was small, but data standardization resulted in their differentiation. From the evaluation results, it can be seen that scheme 2 directly supplied power from generator node 23 to node 24 , with the smallest electrical distance, the highest score for N2-N5 indices, and the highest final comprehensive evaluation result. Therefore, scheme 2 is recommended as the main network transfer scheme.

\section{Conclusions}

In this paper, the V2G capability of an EV cluster was considered, and the power supply paths were searched and evaluated scientifically. First, the mobile behavior of EVs was analyzed on the basis of parking generation rate, and the spatiotemporal characteristics of their discharge capacity was modeled. Then, inter- and intra-station BFS was adopted to realize the intra-system path search, while the edge matching algorithm was used to realize the integrated power supply path search of the main and distribution networks. Lastly, the optimal combination weight was adopted to comprehensively evaluate the paths from multiple angles. According to the example verification in a typical scenario, it was proven that the load transfer strategy proposed in this paper can effectively provide a reference for power grid dispatchers and improve the security and economy of the power system.

The main conclusions are as follows:

- During the working day, the load in the industrial area is high, and the EV discharge capacity in this area is also high. Therefore, during load transfer, part of the load can be transferred to the EV cluster node. The calculation results show that this scheme has a higher score and can be recommended to the dispatchers first.

- In the daytime of non-working days, the load in the commercial area is high, and the discharge capacity of EVs in this area is low; thus, part of the load can be transferred to other transformers or EV cluster nodes in other areas.

- In the evening, the load in residential areas and the discharge capacity of EVs are high; therefore, part of the load can be transferred to the EV cluster node locally to achieve the purpose of peak shaving and avoid the transfer of load to other areas, resulting in network congestion.

Author Contributions: Conceptualization, D.M. and Z.C.; methodology, D.M. and Z.Y.; software, D.M.; investigation, Y.L. and J.T.; resources, X.H.; writing—original draft preparation, D.M.; writingreview and editing, Z.C.; supervision, X.H. All authors have read and agreed to the published version of the manuscript.

Funding: This research was funded by the State Grid Corporation Science and Technology Project (Urban power grid dispatching for large-scale electric vehicle access technical study; 5108-202118041A-0-0-00).

Institutional Review Board Statement: Ethical review and approval were waived for this study since it did not involve humans or animals.

Informed Consent Statement: This study did not involve humans.

Data Availability Statement: The data presented in this study are available from the corresponding author upon request. The data are not publicly available due to them being a commercial secret.

Conflicts of Interest: Yang Lv and Jiang Tian are employees of the State Grid Jiangsu Electric Power Co., Ltd. The paper reflects the views of the scientists, and not the company. 


\section{References}

1. Gong, C.; Zhang, B.; Ding, Y.; Ma, L.; Zhao, Y.; Li, X. Operating Characteristics and Influence on Power Grid with Distributed Electric Heating Considering Load Transfer. In Proceedings of the 2nd IEEE Conference on Energy Internet and Energy System Integration (EI2), Beijing, China, 20-22 October 2018; pp. 1-4.

2. Kleinberg, M.R.; Miu, K.; Chian, H.-D. Improving Service Restoration of Power Distribution Systems Through Load Curtailment of In-Service Customers. IEEE Trans. Power Syst. 2011, 26, 1110-1117. [CrossRef]

3. Azeem, M.Q.; Rehman, H.U.; Ahmed, S.; Khattak, A. Design and analysis of switching in automatic transfer switch for load transfer. In Proceedings of the 2016 International Conference on Open Source Systems \& Technologies (ICOSST), Lahore, Pakistan, 15-17 December 2016; pp. 129-134.

4. May Marma, H.U.; Liang, X. Composite Load Model and Transfer Function Based Load Model for High Motor Composition Load. In Proceedings of the 2019 IEEE Electrical Power and Energy Conference (EPEC), Montreal, QC, Canada, 16-18 October 2019; pp. 1-5.

5. Xu, Z.; Bai, X.; Wang, J.; Liu, M.; Zhang, X. The Control Method of Force Loading of Robot on Load Transfer Mechanism of Space Station. In Proceedings of the 2019 IEEE 15th International Conference on Control and Automation (ICCA), Edinburgh, UK, 16-19 July 2019; pp. 518-523.

6. Xianchao, H.; Taylor, G. Service Restoration of Distribution Networks Based on Node-depth Encoding Technique. Autom. Electr. Power Syst. 2011, 356, 40-44.

7. Gaihong, C.; Zheng, X. Optimal load restoration algorithm based on particle swarm optimization. Autom. Electr. Power Syst. 2007, $16,62-65$.

8. Xiuyi, L.; Chengfeng, Y.; Qingshan, X. Research on Reconfiguration Algorithm of distribution network fault recovery and load balance. Power Syst. Technol. 2002, 267, 34-37.

9. Xueping, G.; Zhonghui, H.; Haiping, L. Optimization algorithm of system partition recovery after power system blackout. Proc. Chin. Soc. Elect. Eng. 2009, 29, 41-46.

10. Ma, C.L.; Ling, W.S.; Wu, J.Y.; Wang, K.L.; Ding, J. Dynamic Load Transfer of Active Distribution Network Based on Prediction Information. In Proceedings of the 2018 China International Conference on Electricity Distribution (CICED), Tianjin, China, 17-19 September 2018; pp. 960-966.

11. Zhong, C.; Yi, L.; Tao, Z.; Qiang, X. Optimal time-of-use charging pricing strategy of EVs considering mobile characteristics. Electr. Power Autom. Equip. 2020, 40, 96-102.

12. Yan, B.; Jiuchun, J.; Weige, Z.; Liyong, N.; Caiping, Z. Model and control strategy of electric vehicle mobile energy storage system. Autom. Electr. Power Syst. 2012, 36, 36-43.

13. Lidan, C.; Rao, Z.; Figueiredo, A. Overview of charging and discharging load forecasting for electric vehicles. Autom. Electr. Power Syst. 2019, 43, 177-191.

14. Bin, Z.; Weiguo, Z.; Wenjia, C.; Dongyu, M.; Zhong, C. Charging and discharging optimization strategy of electric vehicle considering early warning load. Electr. Power Constr. 2020, 41, 22-29.

15. Mingyang, L.; Bin, Z. Charging and discharging decision-making model of electric vehicles and influence analysis of electricity price. Autom. Electr. Power Syst. 2015, 39, 75-81.

16. Long, Y. Research on Load Transfer of Power System Distribution Network. Master's Thesis, Tianjin University, Tianjin, China, 2012.

17. Zheng, S. Research on Load Transfer Capability of Distribution Network. Master's Thesis, Southeast University, Nanjing, China, 2018. 